\title{
Role of Duplex Ultrasound for the Assessment of Malignancy in Breast Tumors
}

\author{
Anindita Rani Paul ${ }^{1}$, Faria Nasreen ${ }^{2}$, Nasreen Sultana ${ }^{2}$ \\ ${ }^{1}$ Institute of Nuclear Medical Physics, Atomic Energy Research Establishment (AERE), Savar, Dhaka, Bangladesh \\ ${ }^{2}$ National Institute of Nuclear Medicine \& Allied Sciences, Bangabandhu Sheikh Mujib Medical University, Shahbag, Dhaka, Bangladesh
}

Email address:

aninditapau1981@gmail.com (A. R. Paul)

\section{To cite this article:}

Anindita Rani Paul, Faria Nasreen, Nasreen Sultana. Role of Duplex Ultrasound for the Assessment of Malignancy in Breast Tumors. Cancer Research Journal. Vol. 7, No. 4, 2019, pp. 143-149. doi: 10.11648/j.crj.20190704.14

Received: September 15, 2019; Accepted: October 14, 2019; Published: October 23, 2019

\begin{abstract}
Background: Breast cancer is the commonest form of cancer in females which may be curable if detected early. Breast ultrasound (US) is a non invasive, primary screening tool which can evaluate breast tumors in women even with dense breast. By adding color Doppler signal with B-mode US namely Duplex ultrasound better characterization of breast tumors is possible especially for the indeterminate tumors (BIRADS category 3 and 4) on B-Mode US. Objective: The purpose of this study is to assess the efficacy of Duplex Sonography (DS) for assessment of malignancy in breast tumors compared to histopathology. Materials and methods: This cross-sectional study was carried out at National Institute of Nuclear Medicine \& Allied Science (NINMAS) from July 2017 to June 2018. A total of 57 female patients having breast tumors (age mean 42.09 years with $\mathrm{SD} \pm 15.85$ ) were included in this study. After clinical assessment, all study patients underwent Duplex Ultrasound and was then subjected to the excisional biopsy and histopathology for confirmation of the diagnosis. Diagnostic performance test of DS was done for diagnosis of breast malignancy compared to histopathology. Receiver-operator characteristic (ROC) curve was also constructed to determine the optimal cut-off point for each spectral Doppler indices. Results: On Color Doppler Sonography (CDS), hypervascularity was more common in malignant tumors than benign lesions $(86.7 \% \mathrm{Vs} 18.5 \%$; $\mathrm{p}=0.001)$. Spectral Doppler indices including resistive index (RI) and pulsatility index (PI) were significantly higher in malignant tumors $(\mathrm{p}=0.001)$. All of these indices had high diagnostic accuracy for differentiating malignant from benign tumors with area under the curve (AUC) which is equals to $0.90,0.92$ for RI and PI respectively. The optimal cut-off points to differentiate benign and malignant tumors from ROC curve for RI was 0.75 (sensitivity: $89.0 \%$, specificity: $85.0 \%$ ) and that of PI was 1.2 (sensitivity: 96.6\%, specificity: $80.0 \%$ ). All obtained results of sensitivity, specificity and over all accuracy of DS (considering the vessel number on CDS \& BIRADS criteria on B-mode US) were $93.3 \%, 81.5 \%$ and $87.7 \%$ respectively. Conversely, conventional BMode US alone had a sensitivity, specificity and over all accuracy of $100 \%, 55.6 \%$, and $78.9 \%$ respectively. Addition of CDS to B-mode US in the same session increased specificity from $55.6 \%$ to $81.5 \%$ and accuracy from $78.9 \%$ to $87.7 \%$. Conclusion: Duplex ultrasound has a significant role in the evaluation of breast tumors and adds value to B-mode US for distinguishing malignant tumors from benign lesions.
\end{abstract}

Keywords: Breast Cancer, Ultrasound (US), Color Doppler Sonography (CDS), Duplex Sonography (DS), Resistive Index (RI), Pulsatility Index (PI)

\section{Introduction}

Breast cancer is the most common cancer in women worldwide with over 2 million new cases in the year 2018 [1] These statistics accentuate the necessity of screening and early diagnosis of breast cancers to decrease mortality [2, 3]. Nowadays, breast US is a widely available, non invasive, standard imaging technique for diagnosing breast tumors in women even with dense breast [3, 4]. Duplex Sonography (DS) of breast is a combination of both conventional B-mode US and CDS. CDS is a modern technique which increases the diagnostic confidence by showing vascularity in breast tumors [3-6]. By adding color Doppler signal better characterization of breast mass is possible specially for those mass with BIRADS category 3 and 4 on B-mode US $[4,7,8]$. Although tumor vascularization is becoming highly 
important in diagnostic, and prognostic terms, there have always been controversies in different studies to define acceptable criteria for differentiating benign and malignant breast lesions by CDS [3, 9]. So the present study was aimed to evaluate the diagnostic accuracy of DS for assessment of malignancy in breast tumors.

\section{Materials and Methods}

This cross-sectional study was carried out at National Institute of Nuclear Medicine \& Allied Science (NINMAS), BSMMU from July 2017 to June 2018. A total of 57 female patients having breast tumors at the age of 15 years or above were included in this study.

\subsection{Methodology}

After obtaining written informed consent, the patients tumors were assessed with both B-mode US and CDS to collect data on pre-formed data sheet. This examination was performed on a Toshiba, Aplio 500 ultrasound machine using a linear, high frequency probe $(10 \mathrm{MHz})$. DS was performed by two fixed, experienced sonologists who were blinded to the pathologic reports of the patients. Subsequently, the patient underwent biopsy within a week after the initial US examination.

\subsection{Technical Consideration}

The color box of CDS was adjusted to include the target lesion with minimal normal surrounding tissue and color gain was set to a level at which low-velocity flow could be identified with minimal background noise. To obtain appropriate images, the transducer had to be applied with a pressure necessary to maintain contact with the skin. The sonologists were careful to apply as little pressure as possible on the transducer to prevent collapsing of the small tumor vessels (less than $0.1 \mathrm{~mm}$ in diameter).

\subsection{Interpretation}

On B-mode US tumors were classified according to BIRADS classification done by American College of Radiology guideline, 2013 [10]. Then number of vessels, RI, PI and vascularization patterns (central or peripheral) were evaluated for all the lesions on Doppler sonography.

Finally, CDS image results were added to B-mode US.

For data analysis purpose the following scoring system was done (Table 1).

For B Mode US:

BIRADS 1, 2 and 3 category tumors were considered as benign and assigned as Score-1.

BIRADS 4 and 5 were considered as malignant and assigned as Score-2.

For CDS:

No vessel and Single vessel were considered as benign and assigned as Score -1 .

When 2 or more vessel were present it was considered as malignant and assigned as Score- 2 .
PSV (Peak Systolic Velocity) and EDV (End Diastolic Velocity) of tumor vessels were measured. RI and PI value were automatically calculated by US machine software and hard copy was generated.

For Duplex US:

A combined Duplex score of 1 was assigned to be benign and 2 was assigned to be malignant.

Table 1. Combined scoring for DS used in this study.

\begin{tabular}{lll}
\hline B-mode score & Color Doppler score & Duplex score \\
\hline 1 & 1 & 1 \\
2 & 2 & 2 \\
1 & 2 & 2 \\
2 (BIRADS 4) & 1 & 1 \\
2 (BIRADS 5) & 1 & 2 \\
\hline
\end{tabular}

\subsection{Statistical Analysis}

Data analyses were carried out by using the Statistical Package for Social Sciences (SPSS) with version 20.0. Statistical diagnostic performance test was used to calculate the efficacy of DS. Sensitivity \& specificity of RI \& PI was presented by ROC curve. In each analysis, level of significance was 0.05 and $P$ value $\leq 0.05$ was considered as significant.

\section{Result}

Duplex ultrasound and exicision biopsy were performed on 57 patients having breast tumor. The mean \pm SD age of study patients was found $42.09 \pm 15.85$ years ranging from 15 82 years (Table 2). Upper outer quadrant (UOQ) was the most commonly involved quadrant $(40.7 \%)$. Regarding the composition of breast tumors, $82.5 \%$ of tumors were solid. Majority $(80.7 \%)$ of them had heterogenous echotexture, $43.9 \%$ had ill-defined or spiculated margins and $22.8 \%$ presented with microcalcification (Table 3 ). Of all the tumors (57), histopathology diagnosed 27 tumors as benign and 30 tumors as malignant. The most common pathology encountered in benign category was fibroadenoma (29.6\%) while the most common pathology in malignant lesions was invasive ductal carcinoma (47.4\%) (Table 4).

Doppler characteristics of all the lesions were evaluated and compared in benign and malignant groups. Most of the malignant tumors $(86.7 \%)$ were hypervascular on CDS. However, hypervascularisation was detected in only $18.5 \%$ of benign masses (Table 5). Among 21 BIRADS 5 category (malignant) tumors 19 tumors had mixed pattern vessels while only two tumors were avascular on CDS (Table 6).

Current study observed that in color Doppler evaluation of a breast tumor, hypervascularity proved to be a reliable sign to predict the possibility of malignancy, as pointed out by various authors previously [4, 6]. In malignant lesions, arteries were arranged in the central part of the lesion with/without vessels in the periphery (Figure 1). Amongst the benign vascular lesions, only five benign lesions showed evidence of hypervascularity (Figure 2). Rest of the vascular benign lesions showed presence of capsular vessels or 1-2 arteries distributed in the peripheral portion of the mass 
(Figure 3).

Spectral Doppler indices including resistive index (RI) and pulsatility index $(P I)$ were significantly higher $(p=0.001)$ in malignant tumors (Table 7). The optimal cut-off points to differentiate benign and malignant tumors from ROC curve for RI (Figure 4) was 0.75 (sensitivity: $89.0 \%$, specificity: $85.0 \%$ ) and that of PI (Figure 5) was 1.2 (sensitivity: 96.6\%, specificity: $80.0 \%$ ).

In the current study, 30 patients were diagnosed as malignant on B-mode US and confirmed by histopathological examination, so there was no false negative diagnosis (Table
8). On DS, 24 cases were diagnosed as benign, but the histopathological examination confirmed it in 22 cases and only two cases were found to be malignant, so there were two false negative diagnosis. Thirty three patients were diagnosed as malignant by DS but the histopathological examination confirmed it in 28 cases and only 5 cases were found to be benign, so there were five false positive diagnosis (Table 9).

Compared to B-mode US with DS, B-mode US had more sensitivity while DS had increased specificity and diagnostic accuracy of the screening B-mode US (Table 10).

Table 2. Age distribution of study participants $(n=57)$.

\begin{tabular}{lll}
\hline & Number of patients & Percentage \\
\hline Age (in years) & & \\
$<30$ & 16 & 28.1 \\
$30-50$ & 30 & 52.6 \\
$>50$ & 11 & 19.3 \\
Mean \pm SD & 42.09 & \pm 15.85 \\
Range (min-max) & 15 & -82 \\
\hline
\end{tabular}

Table 3. Summary of ultrasound findings on B-mode ( $n=57)$.

\begin{tabular}{|c|c|c|}
\hline & Number of patients & Percentage \\
\hline \multicolumn{3}{|l|}{ Breast involved } \\
\hline Left & 28 & 55.5 \\
\hline Right & 29 & 44.4 \\
\hline \multicolumn{3}{|l|}{ Quadrant Involved } \\
\hline UOQ & 34 & 40.7 \\
\hline UIQ & 11 & 33.3 \\
\hline LOQ & 6 & 18.5 \\
\hline LIQ & 6 & 7.4 \\
\hline \multicolumn{3}{|l|}{ Tumor size (mm) } \\
\hline $10-30$ & 34 & 51.9 \\
\hline $31-50$ & 20 & 40.7 \\
\hline$>50$ & 3 & 7.4 \\
\hline Mean \pm SD & 28.82 & \pm 11.61 \\
\hline Range (min-max) & 10 & -68 \\
\hline \multicolumn{3}{|c|}{ Enlarged axillary lymph node } \\
\hline Present & 26 & 45.6 \\
\hline Absent & 31 & 54.4 \\
\hline \multicolumn{3}{|l|}{ Composition } \\
\hline Solid & 47 & 82.5 \\
\hline Cystic & 1 & 1.8 \\
\hline Mixed & 9 & 15.8 \\
\hline \multicolumn{3}{|l|}{ Echo texture } \\
\hline Homogenous & 11 & 19.3 \\
\hline Heterogenous & 46 & 80.7 \\
\hline \multicolumn{3}{|l|}{ Margins } \\
\hline Well defined & 13 & 22.8 \\
\hline Ill defined / spiculated & 25 & 43.9 \\
\hline Microlobulated & 19 & 33.3 \\
\hline \multicolumn{3}{|l|}{ Calcification } \\
\hline Micro calcification & 13 & 22.8 \\
\hline Macro calcification & 4 & 7.0 \\
\hline Absent & 40 & 70.2 \\
\hline
\end{tabular}

Table 4. Histopathological type of tumors among study population ( $n=57)$.

\begin{tabular}{lll}
\hline Type & Number of patients & Percentage \\
\hline Benign & & \\
Fibroadenoma & 17 & 29.6 \\
Fibrocystic disease & 6 & 10.5 \\
Complex cyst & 3 & 5.3 \\
\hline
\end{tabular}




\begin{tabular}{lll}
\hline Type & Number of patients & Percentage \\
\hline Intraductalpapiloma & 1 & 1.8 \\
Phyllodes tumor & 1 & 1.8 \\
Malignant & & \\
Invasive ductal carcinoma & 27 & 47.4 \\
Invasive lobular carcinoma & 1 & 1.8 \\
Mucinous cell carcinoma & 1 & 1.8 \\
\hline
\end{tabular}

Table 5. Degree of vascularity of benign and malignant tumors on Color Doppler Sonography (CDS) ( $n=57)$.

\begin{tabular}{lllllll}
\hline Findings & Total no & $\begin{array}{l}\text { Avascular (no vessel) on } \\
\text { CDS }\end{array}$ & $\begin{array}{l}\text { Hypo vascular (single } \\
\text { vessel) on CDS }\end{array}$ & $\begin{array}{l}\text { Hyper Vascular (2 or } \\
\text { more vessel) on CDS }\end{array}$ & Chi-square test (df) & P value \\
\hline Benign & 27 & $7(25.9 \%)$ & $15(55.6 \%)$ & $5(18.5 \%)$ & 27.99 & $0.001 \mathrm{~s}$ \\
Malignant & 30 & $3(10.0 \%)$ & $1(3.3 \%)$ & $26(86.7 \%)$ & 2 \\
\hline
\end{tabular}

$\mathrm{s}=$ significant

Table 6. Pattern of vascularity on CDS in the BIRADS classified tumors compared to histopathology.

\begin{tabular}{lllllll}
\hline \multirow{2}{*}{ Color Doppler US (CDS) } & BIRADS 2 & & BIRADS 3 & & BIRADS 4 \\
\cline { 2 - 7 } & Benign & Malignant & Benign & Malignant & Benign & Malignant \\
\hline Avascular & 2 & 0 & 3 & 0 & 2 & 1 \\
Vessel in rim/single capsular vessel & 4 & 0 & 5 & 0 & 6 & 1 \\
Both central and peripheral vessels (mixed) & 0 & 0 & 1 & 0 & 4 & 7 \\
Total & 6 & 0 & 9 & 0 & 12 & 9 \\
\hline
\end{tabular}

Table 6. Continued.

\begin{tabular}{lllll}
\hline \multirow{2}{*}{ Color Doppler US (CDS) } & BIRADS 5 & & \multirow{2}{*}{ Total no of benign findings } & \multirow{2}{*}{ Total no of malignant findings } \\
\cline { 2 - 3 } & Benign & Malignant & 7 & 3 \\
\hline Avascular & 0 & 2 & 15 & 1 \\
Vessel in rim/single capsular vessel & 0 & 0 & 5 & 26 \\
Both central and peripheral vessels (mixed) & 0 & 19 & 27 & 30 \\
Total & 0 & 21 & & \\
\hline
\end{tabular}

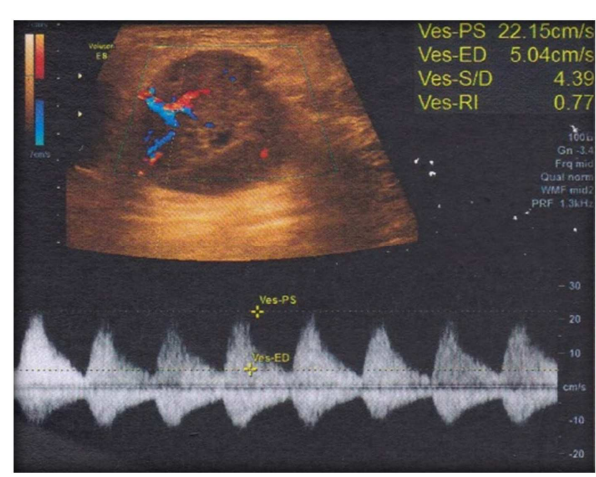

Figure 1. A 48 years old female patient with invasive ductal carcinoma of the breast showing a hypoechoic mass with irregular margin. On color Doppler showing presence of hypervascularity with RI 0.77 which is in favour of malignancy.

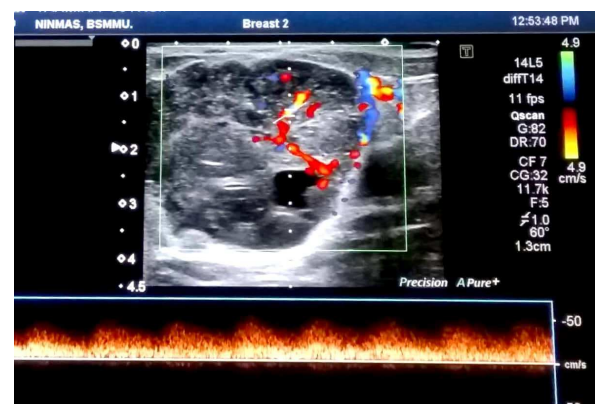

Figure 2. A 50 years old female patients with benign phyllodes tumors of breast showing presence ofhypervascularity on $C D S$.

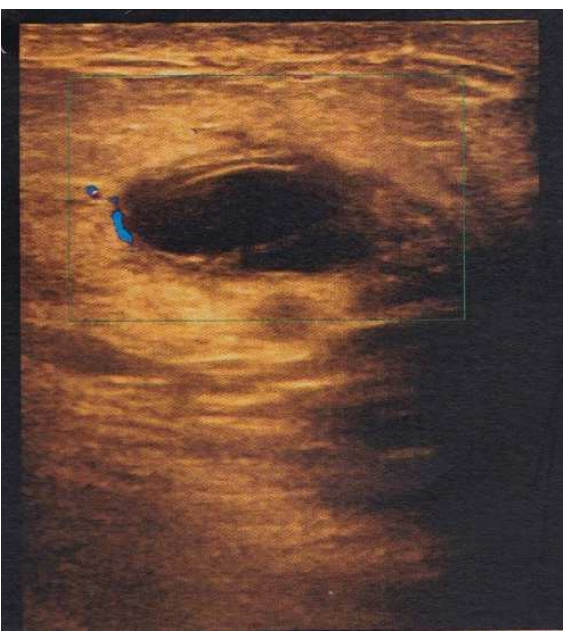

Figure 3. A 28 years old female patient with benign tumor of breast (BIRADS-2) showing presence of vessel in the periphery on CDS.

Table 7. Vascular index of benign and malignant tumors.

\begin{tabular}{llllll}
\hline \multirow{2}{*}{ Vascular index } & \multicolumn{2}{l}{$\begin{array}{l}\text { Benigntumors } \\
(\mathbf{n}=\mathbf{2 7})\end{array}$} & $\begin{array}{l}\text { Malignant tumors } \\
(\mathbf{n}=\mathbf{3 0})\end{array}$ & \multirow{2}{*}{ P value } \\
\cline { 2 - 5 } & Mean & \pm SD & Mean & \pm SD & \\
\hline RI & 0.61 & \pm 0.15 & 0.83 & \pm 0.13 & $0.001^{\mathrm{s}}$ \\
Range (min-max) & 0.28 & -0.91 & 0.6 & -1.3 & \\
PI & 1.05 & \pm 0.51 & 2.17 & \pm 0.68 & $0.001^{\mathrm{s}}$ \\
Range (min-max) & 0.12 & -2.4 & 1.06 & -3.86 & \\
\hline
\end{tabular}

$\mathrm{s}=$ significant

$\mathrm{p}$ value reached from unpaired t-test 


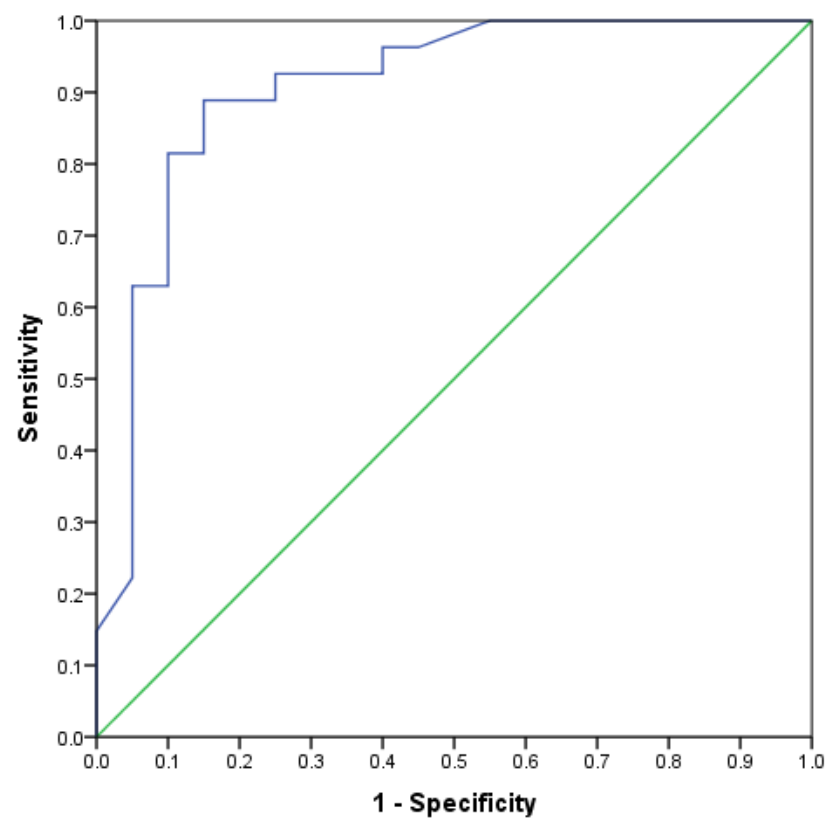

Diagonal segments are produced by ties.

Figure 4. Receiver operating characteristic (ROC) curve of RI had a area under the curve (AUC 0.90) that means this value is significantly associated with detection of breast malignancy.

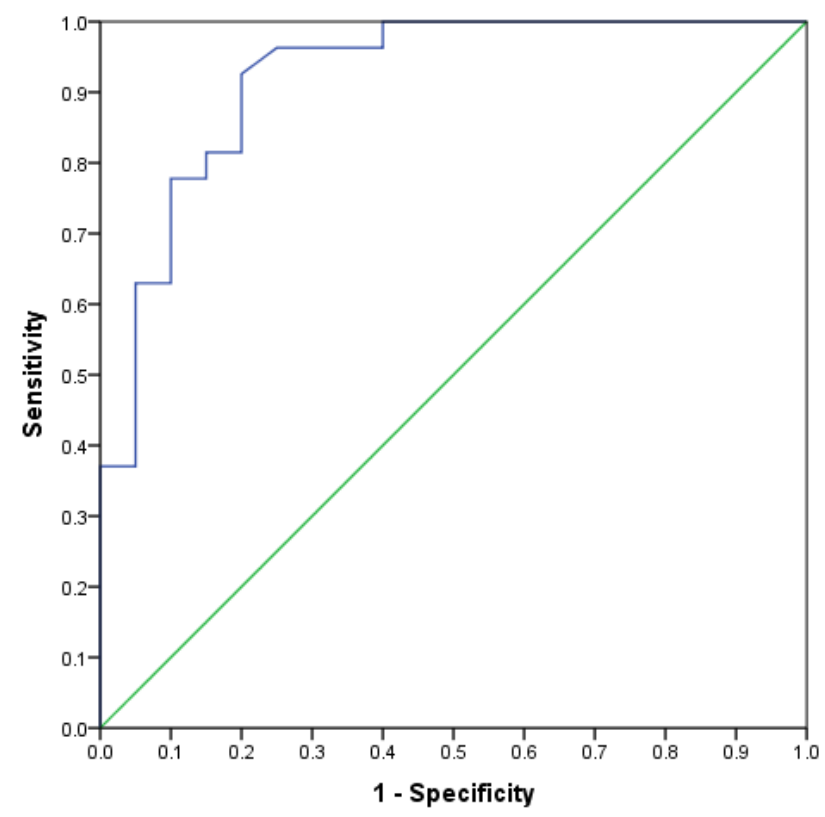

Diagonal segments are produced by ties.

Figure 5. Receiver operating characteristic (ROC) curve of PI had a good area under the curve (AUC 0.92) which is significantly associated with detection of breast malignancy.

Table 8. Performance of B-mode ultrasound for diagnosis of breast malignancy compared to histopathology.

\begin{tabular}{|c|c|c|c|}
\hline \multirow{2}{*}{ B-Mode US } & \multicolumn{2}{|c|}{ Histopathology } & \multirow{2}{*}{ Total } \\
\hline & Malignant & Benign & \\
\hline Malignant & $30(100.0)$ & $12(44.4)$ & $42(73.7)$ \\
\hline Benign & $0(0.0)$ & $15(55.5)$ & $15(26.3)$ \\
\hline Total & $30(100.0)$ & $27(100.0)$ & $57(100.0)$ \\
\hline
\end{tabular}

(Figure within parentheses indicates in percentage)

Table 9. Performance of Duplex Ultrasound for diagnosis of breast malignancy compared to histopathology.

\begin{tabular}{|c|c|c|c|}
\hline \multirow{2}{*}{ Duplex US (Combined B-mode US \&CDS) } & \multicolumn{2}{|c|}{ Histopathology } & \multirow{2}{*}{ Total } \\
\hline & Malignant & Benign & \\
\hline Malignant & $28(93.3)$ & $5(18.5)$ & $33(57.9)$ \\
\hline Benign & $2(6.6)$ & $22(81.5)$ & $24(42.1)$ \\
\hline Total & $30(100.0)$ & $27(100.0)$ & $57(100.0)$ \\
\hline
\end{tabular}

(Figure within parentheses indicates in percentage)

Table 10. Comparison of performance between B-mode US and Duplex Ultrasound.

\begin{tabular}{lll}
\hline Validity test & B-Mode US & Duplex Ultrasound (Combined B-Mode \& CDS) \\
\hline Sensitivity & 100.0 & 93.3 \\
Specificity & 55.6 & 81.5 \\
Accuracy & 78.9 & 87.7 \\
Positive predictive value & 71.4 & 84.8 \\
Negative predictive value & 100.0 & 91.7 \\
\hline
\end{tabular}

\section{Discussion}

Ultrasound is a more sensitive (95\% sensitivity) diagnostic test than mammogram ( $85 \%$ sensitivity) for detection of breast cancer in younger female with dense breast [4]. CDS is an adjunct tool to B-mode US imaging findings in differentiating malignant from benign tumor of breast $[4,6$, 11-14]. Malignant tumors show increased vascularity due to neoangiogenesis $[4,11]$. Many authors suggest that a mass with central vessels and lack of capsular vessels, penetrating vessels entering the mass directly are suspicious for malignancy [4-6, 12].

Color Doppler analysis of this study revealed that the majority of the malignant masses were hypervascular $(86.7 \%)$ with peripheral and central vascularity, while the benign lesions were predominantly avascular $(25.9 \%)$ or hypovascular $(55.6 \%)$. Ten percent of malignant lesions in 
this study were avascular on CDS (Table 5), which is similar to the findings of a study by Gupta et al. [4]. The avascularity of malignant tumors may be due to very small caliber vessels within the mass or low grade low cellularity tumors or desmoplastic change of the tumors [9]. However, some benign tumors can be hypervascular $[4,6]$. In this study, we found $18.5 \%$ hypervascular benign tumors (Table 5). This is not unusual and makes it difficult to differentiate malignant tumors from benign lesions just based on color Doppler pattern [13].

In the present study, the mean value of RI and PI were significantly higher in malignant tumors (Table 7) which correlate with some previous studies [3-5, 13]. They suggest that a high RI increases the chances of malignancy in a breast mass; mainly related to the presence of areas of stenosis, occlusions, great increase of peripheral resistance of vascular bed of some tumors and arteriovenous shunts and lack of smooth muscle or abnormal structure of vessels in malignant tumors [4].

Current study found a RI value higher than 0.75 had sensitivity and specificity value of $89.0 \%$ and $85.0 \%$ respectively, as a sign of malignancy (Figure 4). A PI value higher than 1.2 as a sign for malignancy had sensitivity equal to $96.6 \%$, and specificity equal to $80.0 \%$ (Figure 5). Several studies have used different RI and PI values as the cut-off points for differentiating benign and malignant breast masses [3, 4, 13]. Studying on 100 breast masses, Keshavarz et al. demonstrated that RI $\geq 0.68 \&$ PI $\geq 0.93$ are significantly predictive of malignancy [13]. Conversely, many authors have reported that the analysis of spectral Doppler criteria such as RI and PI values contribute less in differentiation between benign and malignant breast masses than color Doppler since there is a wide overlap of spectral parameters [4, 5, 11]. So, further investigation with large sample size is necessary to reach acceptable cut off points for PI and RI.

The value of any diagnostic test lies in it's ability to detect the presence of disease when it is present (sensitivity) and verify the absence of disease when it is not present (specificity) [14]. In this study, for calculating the performance of Duplex Sonography only the number of vessels were taken into account in the CDS along with the Bmode findings. On the basis of the above mentioned criteria the calculated sensitivity, specificity, accuracy, PPV, NPV of DS were $93.3 \%, 81.5 \%, 87.7 \%, 84.8 \%$ and $91.7 \%$ respectively (Tables 9 and 10). When comparing between Bmode US and DS most studies have reported better specificity for DS and higher sensitivity for B-mode US (Table 10) which is similar to this study results $[7,8,15,16]$. The lower specificity of B-mode US compared to DS in this study was due to a total of $12(44.4 \%)$ false positive cases (Table 8). The major sources of false-positive findings at Bmode screening US [12 of 57] in this study were the BIRADS category 3 and 4 tumors which were also the most frequent findings at B-mode US as suggested by Lee et al [7].

On the other hand, on CDS only five benign hypervascular (false positive) tumors in this study decreased the specificity of DS. Among four false negative malignant tumors on CDS three were avascular, one was hypovascular. Among these four malignant Doppler negative tumors two were BIRADS category 4 on B-mode US that reduced the sensitivity of DS (Table 6). Although two BIRADS 5 category tumors were avascular (Doppler negative) but on Duplex scoring they were considered as malignant according to the study of Lee et al. [7]. So for those BIRADS 5 tumors the Duplex ultrasound did not give false negative result. By applying Doppler signal with B-mode US, higher specificity and diagnostic accuracy can be achieved as also suggested by previous study [14-16].

\section{Conclusion}

The combined use of CDS with B-mode US in the same session increased diagnostic performance (Specificity \& Accuracy) of B-mode US in distinguishing benign from malignant breast masses. A considerable number of falsepositive findings can be reduced by selective downgrading of BI-RADS category 3 and 4 masses to category 2 that show results negative for cancer at color Doppler scoring in asymptomatic women. However, color Doppler findings cannot be used in isolation as a standalone predictor of malignancy as malignant tumors can be avascular. Furthermore, RI, PI, and pattern of vascularization have a good ability to differentiate and predict the nature of breast lesions. As DS provide useful information about the nature and prognosis of a mass these should be used routinely to assess and evaluate all breast tumors.

\section{Limitations}

This present study had few limitations. Firstly, the sample size is relatively small due to reasons such as refusal by patients for breast biopsies to be taken. Secondly, CDS is highly operator and equipment-dependent, inducing interobserver variability. As CDS was not conducted by a single sonologist, interobserver variability may affect the result.

\section{References}

[1] Bray F, Ferlay J, Soerjomataram I, Siegel RL, Torre LA, Jemal A. Global Cancer Statistics 2018: GLOBOCAN estimates of incidence and mortality worldwide for 36 cancers in 185 countries. CA: ACancer Journal for Clinicians. Vol. 68, No. 6, 2018, pp. 394-424. DOI: 10.3322/caac.21492.

[2] Kopans DB. The 2009 U.S. Preventive Services Task Force guidelines ignore important scientific evidence and should be revised or withdrawn. Radiology. Vol. 256, No. 1, 2010, pp. 15-20. DOI: 10.1148/radiol.10100057.

[3] Sirous M, Sirous R, Nejad FK, Rabeie E, Mansouri M. Evaluation of different aspects of power Doppler sonography in differentiating and prognostication of breast masses. Journal of research in medical sciences. Vol. 20, No. 2, 2015, pp. 133-139. 
[4] Gupta K, Chandra T, Kumaresan M, Venkatesan B, Patil AB. Role of color Doppler for assessment of malignancy in solid breast masses: a prospective study. International Journal of Anatomy, Radiology and Surgery. Vol. 6, No. 1, 2017, pp. 5965. DOI: 10.7860/IJARS/2017/24787:2246.

[5] Stanzani D, Chala LF, Barros ND, Cerri GG, Chammas MC. Can Doppler or contrast-enhanced ultrasound analysis add diagnostically important information about the nature of breast lesions? Clinics (Sao Paulo). Vol. 69, No. 2, 2014, pp. 87-92. DOI: $10.6061 /$ clinics/2014(02)03.

[6] Horvath D, Cuitiño M, Pinochet M, Sanhueza P. Color Doppler in the study of the breast: How do we perform it? Revista Chilena de radiologia. Vol. 17, No. 1, 2011, pp. 1927.

[7] Lee SH, Chung J, Choi HY, Choi SH, Ryu EB, Ko KH et al. Evaluation of screening US-detected breast masses by combined use of elastography and color Doppler US with Bmode US in women with dense breasts: a multicenter prospective study. Radiology. Vol. 285, No. 2, 2017, pp. 660669. DOI: $10.1148 /$ radiol.2017162424

[8] Cho N, Jang M, Lyou CY, Park JS, Choi HY, Moon WK. Distinguishing benign from malignant masses at breast US: combined US elastography and color Doppler US - influence on radiologist accuracy. Radiology. Vol. 262, No. 1, 2012, pp. 80-90. DOI: 10.1148/radiol.11110886.

[9] Busilacchi P, Draghi F, Preda L, Ferranti C. Has color Doppler a role in the evaluation of mammary lesions? Journal of Ultrasound. Vol. 15, No. 2, 2012, pp. 93-98. DOI: 10.1016/j.jus.2012.02.007

[10] Zonderland H, Smithuis R. BI-RADS for mammography and ultrasound 2013 updated version. Radiology Assistant. 2014.
[11] Davoudi Y, Borhani B, Rad MP, Matin N. The role of Doppler sonography in distinguishing malignant from benign breast lesions. Journal of Medical Ultrasound. Vol. 22, No. 2, 2014, pp. 92-95. DOI: 10.1016/j.jmu.2013.12.001.

[12] Lee SW, Choi HY, Baek SY, Lim SM. Role of color and power Doppler imaging in differentiating between malignant and benign solid breast masses. Journal Clinical Ultrasound. Vol. 30, No. 8, 2002, pp. 459-64. DOI: 10.1002/jcu.10100.

[13] Keshavarz E, Mehrjardi MZ, Karimi MA, Valian N, Kalantari M, Valian K. Diagnostic value of spectral Doppler ultrasound in detecting breast malignancies. International Journal of Cancer Management. Vol. 11, No. 2, 2018. DOI: $10.5812 / \mathrm{ijcm} .8200$.

[14] Zaini H. Role of color Doppler ultrasound versus histopathology in differentiating malignant from benign breast masses. Iraqi Academic Scientific Journal. Vol. 5, No. 2, 2006, pp. 155-159.

[15] Li L, Zhou X, Zhao X, Hao S, Yao J, Zhong W, Zhi H. BMode ultrasound combined with color Doppler and strain elastography in the diagnosis of non-mass breast lesions: A prospective study. Ultrasoun in Medicine \& Biology. Vol. 43, No. 11, 2017, pp. 2582-2590. DOI: 10.1016/j.ultrasmedbio.2017.07.014.

[16] Choi JS, Han BK, Ko EY, Ko ES, Shin JH, Kim GR. Additional diagnostic value of shear-wave elastography and color Doppler US for evaluation of breast non-mass lesions detected at B-mode US. Eur Radiol. Vol. 26, No. 10, 2016, pp. 3542-3549. DOI: 10.1007/s00330-015-4201-6. 